\title{
POSTOPERATIVE ANALGESIA. OUR SIX MONTHS EXPERIENCE OF ACUTE POSTOPERATIVE PAIN UNIT
}

Vila M, Sifontes K, O`Farrill G, Román M, García-Miguel FJ. General Hospital of Segovia, Spain.

\section{INTRODUCTION}

The treatment of acute pain, especially in postoperative (DAP), is a management challenge. A proper coordination between health professionals, clinical services, and managers is needed. For that reason, the Acute Pain Units (APU) are created, which involves the anaesthesiologist

in the patient care once he is transferred to another service. In this study, we outline the experience in General Hospital of Segovia during the first year of operation of the APU in the

\section{OBJECTIVE}

1. To describe the patients who received continuous epidural analgesia from November 1 , 2014, to April 30, 2015.

2. To study the clinic and demographic characteristics of the target population.

3. To study the quality of analgesia achieved associated symptoms and complications.

\section{METHODS}

Descriptive Transversal Study, the sample of 117 patients with continuous epidural analgesia for DAP control for scheduled surgery during a period of November 1,2014, to April 30, 2015, in General Hospital of Segovia. Instrument: data collection sheet (fig. 1). Includes demographics variables (fig. 2) and pain associated variables (visual analgesic scale and Bromage)

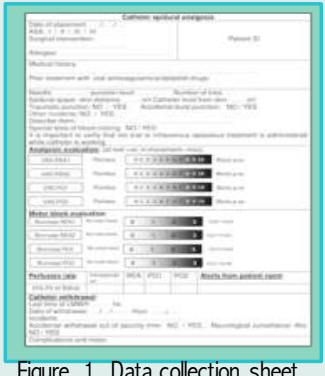

Figure 1. Data collection sheet

CONCLUSION
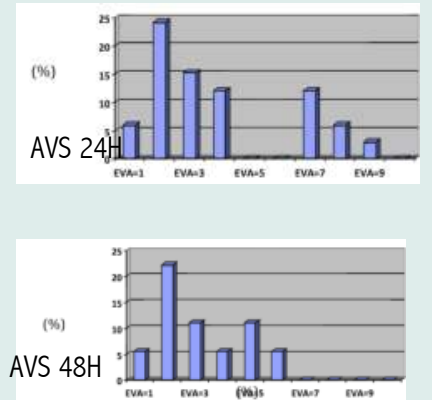

\begin{tabular}{|c|c|c|}
\hline VARIABLES & $\begin{array}{c}\text { WOMEN } \\
n=12(10,3 \%)\end{array}$ & $\begin{array}{c}\text { MEN } \\
n=103(89,7 \%)\end{array}$ \\
\hline AGE & $\begin{array}{c}61,8 \pm 6,6 \\
\text { Mode } 59(19-89)\end{array}$ & $\begin{array}{c}68,7 \pm 5,9 \\
\text { Mode } 71(16-88)\end{array}$ \\
\hline $\begin{array}{c}\text { ASA classification } \\
\text { I } \\
\text { II } \\
\text { III } \\
\text { IV }\end{array}$ & $\begin{array}{c}2(16,7 \%) \\
8(66,7 \%) \\
2(16,7 \%) \\
-\end{array}$ & $\begin{array}{c}19(18,4 \%) \\
65(63,1 \%) \\
17(16,5 \%) \\
2(1,9 \%)\end{array}$ \\
\hline $\begin{array}{c}\text { SURGICAL } \\
\text { SERVICE } \\
\text { General surgery } \\
\text { Uro } \\
\text { Trauma }\end{array}$ & $\begin{array}{c}5(41,7 \%) \\
6(50 \%) \\
1(8,3 \%)\end{array}$ & $\begin{array}{c}43(40,8 \%) \\
41(39 \%) \\
21(20 \%)\end{array}$ \\
\hline $\begin{array}{c}\text { CV RISK FACTORS } \\
\text { YES } \\
\text { NO }\end{array}$ & $\begin{array}{c}2(16,7 \%) \\
10(83,3 \%)\end{array}$ & $\begin{array}{l}64(62,1 \%) \\
39(37,9 \%)\end{array}$ \\
\hline $\begin{array}{l}\text { CATHETER } \\
\text { POSITION } \\
\text { LUMBAR } \\
\text { THORACIC }\end{array}$ & $\begin{array}{l}10(83,3 \%) \\
2(16,7 \%)\end{array}$ & $\begin{array}{l}83(79,8 \%) \\
21(20,2 \%)\end{array}$ \\
\hline
\end{tabular}

Figure 2. Dwmographic variables

In our experience, the continuous epidural analgesia has been proven to be an effective treatment for acute postoperative pain. Although non-serious side effects appeared in $26 \%$ of patients, they were all controlled without further complications. A qualified team with protocols well designed to develop an effective Acute Postoperative Pain Unit was required. 